\title{
Micro- and nanoscale characterization of different natural biomaterials for ocular surface regeneration
}

\section{Gamze Dereli Can ${ }^{1} \odot$, Atakan Tevlek ${ }^{2} \odot$, Mehmet Erol Can $^{1} \odot$, Elif Öncï ${ }^{3} \odot$, Halil Murat Aydın $^{4} \odot$, Nurullah Çağıl ${ }^{5} \odot$}

\author{
${ }^{1}$ Department of Ophthalmology, University of Health Sciences, Bursa Yüksek Ihtisas Training and Research Hospital, Bursa, Turkey \\ ${ }^{2}$ Department of Bioengineering, Hacettepe University, Institute of Science and Engineering, Ankara, Turkey \\ ${ }^{3}$ Department of Periodontology, Necmettin Erbakan University Faculty of Dentistry, Konya, Turkey \\ ${ }^{4}$ Department of Environmental Engineering, Hacettepe University, Bioengineering Division and Centre for Bioengineering, Ankara, \\ Turkey \\ ${ }^{5}$ Department of Ophthalmology, Ylldırım Beyazıt University School of Medicine, Ankara, Turkey
}

\section{ABSTRACT}

Objectives: This study aims to characterize the widely used biological derived membranes in clinics in terms of micro-nano scale mechanical and morphological properties. Within this scope, advanced platelet-rich fibrin (A-PRF), leucocyte-and platelet-rich fibrin (L-PRF) and human amniotic membrane were studied in this research study.

Methods: Nano-indentation, optical coherence tomography (OCT), scanning electron microscopy (SEM), and in vitro degradation test were performed for material characterization.

Results: The nano-indentation test revealed significantly higher modulus of elasticity and hardness values in A-PRF group, while OCT presented significantly higher thickness measurements when compared L-PRF. A loose $3 \mathrm{D}$ architecture formation due to the large pores formed by means of large fiber diameter were observed in A-PRF group. Besides, platelets were observed among the large fibers in A-PRF membranes on the contrary of L-PRF membranes. Low fiber diameter and high cellular separation were recorded in L-PRF group due to the high centrifugal force application. Therefore, it was observed that the platelets were located mostly on the surface of the membranes in L-PRF. The loose 3D architecture of A-PRF membranes is thought to release growth factors for a longer period of time, ensuring cellular integrity. On the other hand, degradation test results indicated that amniotic membranes degrade to about $85 \%$ in one week, while L-PRF and A-PRF were lost their initial weights approximately $31 \%$ and $40 \%$, respectively.

Conclusions: This comparative characterization study of three different natural biomaterials used in a wide range of clinical applications, from dentistry to ophthalmology, was thought to guide surgeons on the selection of site-specific material.

Keywords: Leucocyte- and platelet-rich fibrin, advanced platelet-rich fibrin, amniotic membrane, nanoindentation, optical coherence tomography

$\mathrm{T}$ issue engineering is an interdisciplinary field, which combines life sciences, engineering techniques, cell biology, and biomaterials science. It tar- gets maintenance, improvement or regeneration of tissue function with modernized approaches including cellular and/or scaffold-based applications [1]. The 
major concern in this field is to obtain the most safe, simple and clinically applicable and also the least harmful and expensive product. Unfortunately, the compatibility, degradability, and versatility of many biomaterials are not satisfactory for desired regeneration of soft and hard tissue defects $[1,2]$. The researchers have therefore focused on blood-derived autologous biomaterials which are important source of essential cellular and protein-based products that cannot be obtained from other synthetic biomaterials [2-5].

Platelet concentrate trapped in 3 dimensional (3D) fibrin architecture is one of the blood derivative products and has gained popularity in the last two decades with major innovations of different preparation protocols [4-8]. Leucocyte- and platelet-rich fibrin (L-PRF), derived from individual's own blood sample by Choukroun's technique, is a member of platelet-rich product family and consists of leukocytes, cytokines, circulating stem cells, matrix proteins andgrowth factors (GFs) within the platelet granules which induce and accelerate tissue regeneration $[6,7]$. Unlike the previously proposed platelet-rich preparation method in the literature, Choukroun's recommendation does not use techniques that prolong the duration of the procedure and have side effects, such as anticoagulants, bovine serum or multi-step centrifugation $[4,6,7]$. When preparing the standard L-PRF, firstly venous blood sample of a patient is obtained by syringe and transferred to anticoagulant-free glass tube in which the blood sample starts to coagulate rapidly when contacted with the tube wall. Following, the tubes provide L-PRF elution when centrifuged at 2,700 rpm for 12 minutes due to its clot activator feature. Primarily, fibrinogen is formed at the top of the tube. The circulating thrombin then transforms the fibrinogen into the fibrin, and this fibrin clot accumulates in the middle of the tube like a buffy coat between the red corpuscles at the bottom and the acellular plasma at the top $[6,7]$. Although the proposed standard L-PRF matrices seem to be a good source of biomaterials for the healing and regeneration process, it is not clear that which kind of size, shape, density of fibrin matrices together with cell distribution in the fibrin architecture will provide the optimized conditions for regeneration. Studies in recent years mostly seeks to determine the optimum centrifugation process to obtain more cellular and GFenriched fibrin matrix that supports GF release to the regeneration side and therefore cell migration $[9,10]$. Ghanaati et al. [9] demonstrated that decrease in centrifugation speed $(1,500 \mathrm{rpm})$ together with increase in centrifugation time (14 minutes) result in higher platelet concentrations and enhanced presence of neutrophilic granulocytes in the distal part of the clot away from the buffy coat. In addition to high platelet and GF concentrations in platelet granules, the presence of neutrophilic granulocytes in the microenvironment contributes to monocyte-macrophage differentiation that resulting in accelerated tissue regeneration due to additional GF release from macrophages. Because of this improved cell and GF composition, the advanced PRF (A-PRF) acronym was assigned for the description of this matrix [9]. Like the Ghanaati's investigation, Kobayashi et al. [10] showed that A-PRF releases significantly higher total quantities of GF throughout a 10-day period and is suitable for conditions that require long-term release. However, in another study, biological features such as GF release and degradation time of standard L-PRF was compared with A-PRF and the released GF from standard L-PRF was found two times higher than A-PRF. In addition, it was observed that A-PRF membranes lost their initial shape by degrading earlier than the L-PRF membranes [11]. Due to the increasing accumulation of confounding results of A-PRF, the idea of evaluating the morphological and mechanical properties of this new matrix has emerged.

Both forms of PRFs have been widely used in clinical approaches by surgeonsdue to their reconstructive properties. Further, recent human and animal studies reported that the PRF enhances surface regeneration by giving rise to epithelial cells to migrate and grow because of its biologically derived scaffold function $[12,13]$. Although the L-PRF's and amniotic membrane's biological, mechanical and morphological features [5, 14-16], and A-PRF's cellular composition and release dynamics [9-11] have been investigated, there have been no studies to investigate the A-PRF's mechanical and morphological properties in the literature.In addition, the lack of comparison between these biomaterials can lead to confusion in pre-determining the appropriate material for the relevant surgical procedure.

The objective of this in vitro study is to elucidate the structure of A-PRF by nanomechanical, morphological and degradation test methods and to compare 
it with autogenous L-PRF and allogenic human amniotic membrane, which is frequently preferred in clinical practice for ocular surface reconstruction.

\section{METHODS}

This experimental study was conducted at Ophthalmology Departments, Yildırım Beyazıt University School of Medicine and Keçioren Training and Research Hospital; and Institute of Science and Engineering, Bioengineering Division, Hacettepe University, Ankara, Turkey, according to the tenets of the declaration of Helsinki. Approval was obtained from the Institutional Review Board of Kecioren Training and Research Hospital (2012-KAEK15/1066) and written informed consent was obtained from all participants after the explanation of the research design.

\section{Test Subjects}

We enrolled a total of 10 healthy volunteers for the obtainment of PRF membranes (PRF group) and 10 healthy, volunteer pregnant femalesunderwent caesarean section for the obtainment of 10 pieces of amniotic membrane (amniotic membrane group). Patients with a history of smoking, anticoagulant medication, systemic disease, contagious diseases including Acquired Immune Deficiency Syndrome, hepatitis and syphilis were excluded. For each individual in the PRF group, $20 \mathrm{ml}$ peripheral venous blood sample was obtained and immediately transferred to centrifugation process. $10 \mathrm{ml}$ of the blood substitutes were applied standard L-PRF preparation protocol (L-PRF group) while the other 10 $\mathrm{ml}$ underwent A-PRF preparation protocol (A-PRF group).

\section{Preparation of L-PRF and A-PRF}

Twenty-ml blood sample wasobtained by a vacutainer needle from the patient's antecubitalvein under sterile conditions. Samples were divided into 2 separate $10 \mathrm{ml}$ glass tubes without anticoagulant and immediately transferred to centrifugation process. In L-PRF group, the blood sample was centrifuged at 2,700 rpm for 12 minutes (standard protocol) using a table centrifuge system (Hettich EBA-20; Hettich Holding GmbH \& Co. oHG, Germany). In A-PRF group, the blood sample was centrifuged at 1,500 rpm for 14 minutes with the same device. The fibrin clots formed between the red blood corpuscles at the bottom and platelet poor plasma at the top were removed (Fig. 1a), and remnants of red blood cells were scraped off and discarded with gauze (Fig. 1b). The clotswere then transferred to a PRF box (Medisoft Medical, Turkey) and compressed to obtain L-PRF and A-PRF membranes (Fig. 1c). All of the membranes were transferred into $\mathrm{pH} 7.4$ phosphate buffer solution (PBS) in sterile transportation tubes.

\section{Preparation of Human Amniotic Membrane}

The placentas, obtained from Caesarean section of

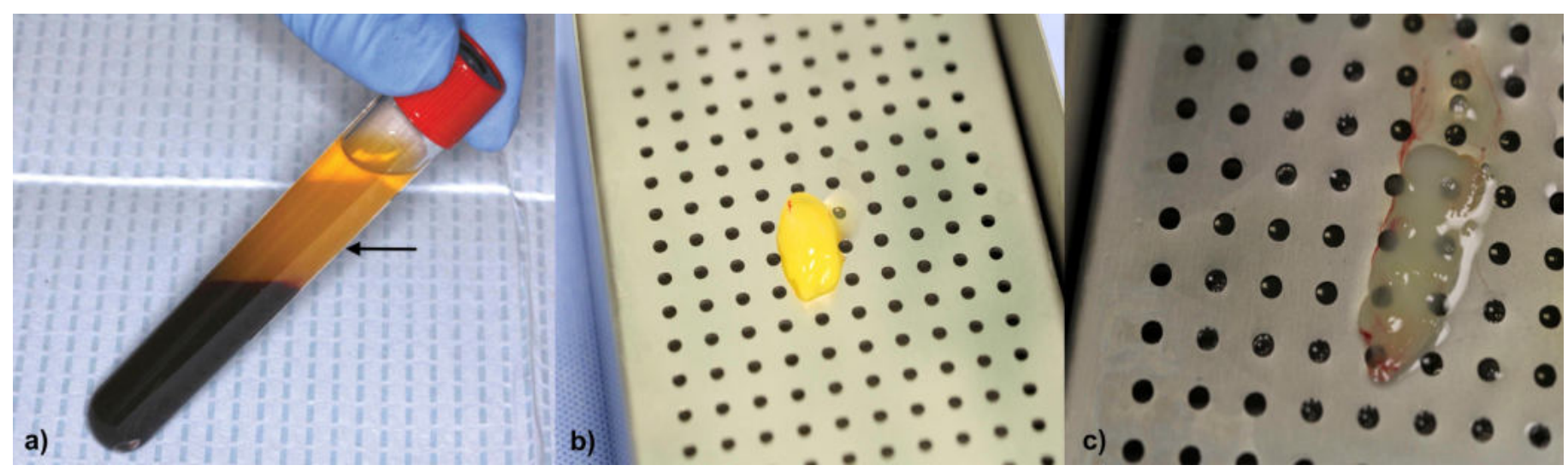

Fig. 1. The preparation of PRF after one-step centrifugation process: a) Fibrin clot was concentrated (arrow) between the red blood cell corpuscule at the bottom and the acellular plasma at the top of the tube, b) Separation of the PRF clot from red blood cell layer, c) Suturable membrane prepared from the fibrin clot by pressing in the PRF membrane box. Note the semi-transparency and smooth surface of the membrane. 
10 females, were cleaned with $\mathrm{pH}$ 7.4 PBS containing $50 \mathrm{mg} / \mathrm{ml}$ penicillin (Sigma-Aldrich, Germany), 50 $\mu \mathrm{g} / \mathrm{ml}$ streptomycin (Sigma-Aldrich, Germany), 100 $\mathrm{mg} / \mathrm{ml}$ neomycin (Biowest, USA) and $2.5 \mathrm{mg} / \mathrm{ml}$ amphotericin B (Hyclone, England) under sterile conditions. The amnion was separated from the chorion by blunt dissection and remnants of chorion were removed by extra-washing with $\mathrm{pH}$ 7.4 PBS.The proximal part of the amniotic membranes were placed on nitrocellulose paper strips within $\mathrm{pH}$ 7.4 PBS in sterile transportation tubes.

\section{Evaluation of Thickness by Spectral Domain Optical Coherence Tomography (SD-OCT)}

The membranes were placed between 2 glass slides without any pressure and these slides were positioned in front of the fixation point of the SD-OCT (Optovue Inc., USA) (regularly calibrated). Images were taken using the anterior segment option (CAML) that provided a radial scan with 12 spaced lines around the buffy coat areain L-PRF and A-PRF groups, and central region in amniotic membrane group by same examiner (GDC).A total of 3 measurements for each membrane were recorded, and the mean thickness values were calculated.

\section{Evaluation of Mechanical Properties by Nano- Indentation}

Nano-indentation studies were conducted to investigate the mechanical properties of the membranes. Membranes were cut into strips of $10 \mathrm{~mm}$ $\times 5 \mathrm{~mm}$ in size including the central buffy coat area in L-PRF and A-PRF groups and central region in amniotic membrane group, and kept in the $\mathrm{pH}$ 7.4 PBS before being subjected to mechanical testing. The modulus of elasticity and hardness values of the membranes were recorded by using a nano-indentation tester (CSM Instrument Nano-indentation Tester, Switzerland) (regularly calibrated) with Olivier Pharr method. It was excavated to a depth of $6 \mu \mathrm{m}$ from the membrane surface and $10 \mathrm{mN}$ load was applied for all samples. Measurements were taken from 5 different points of the membrane surface and mean values were calculated together with standard deviation.

\section{Evaluation of Morphology by Scanning Electron Microscope (SEM)}

Structure and surface morphology of the membranes were examined using SEM (Supra 50VP, Germany) (regularly calibrated). Samples were first cut into strips of $10 \mathrm{~mm} \times 5 \mathrm{~mm}$ in size including the central buffy coat area and distal area separately in L$\mathrm{PRF}$ and A-PRF groupsand central region in amniotic membrane group, then fixed with $2.5 \%$ glutaraldehyde (Sigma Aldrich, Germany) and subjected to series of increasing concentrations of alcohol. In order to remove the water inside the samples completely, hexamethyldisilazane (Sigma Aldrich, Germany) was applied. Finally, the materials were sputter-coated with gold-palladium alloy before SEM imaging. Operated voltage was set at $20 \mathrm{kV}$ and images were obtained from the samples at different magnifications.Fiber diameters were calculated by an image analysis program (Image J; National Institutes of Health, USA) through the obtained photographs.

\section{In Vitro Degradation}

Samples from each experimental group were cut into strips of $10 \mathrm{~mm} \times 5 \mathrm{~mm}$ in size. In order to obtain the dry weight of the membranes, samples were placed in an incubator set to $37^{\circ} \mathrm{C}$ for overnight. Initially, the membranes were weighed on an electronic micro weighing scale (Denver Instrument, Germany) (regularly calibrated). When they reached at constant weight, each sample was immersed inglass vials containing $10 \mathrm{ml} \mathrm{pH} \mathrm{7.4} \mathrm{PBS} \mathrm{supplemented} \mathrm{with} \mathrm{1 \%}$ $\mathrm{v} / \mathrm{v}$ penicillin-amphotericin B.The glass vials containing samples were fixed on a shaker set (Gerhardt, Germany) set to $60 \mathrm{rpm}, 37^{\circ} \mathrm{C}$ and the degradation medium was changed daily. At the end of 1-week, the membranes were taken out of the incubation medium, washed with distilled water, dried, and weighed. The degradation results were given as a graph in comparison with the initial dry weight.

\section{Statistical Analysis}

Statistical analysis was performed by using the Statistical Package for Social Sciences software version 20.0 (SPSS Inc, Chicago, IL, USA).All quantitative data was presented as mean \pm standard deviation for all groups. Significance of difference in the variables was assessed by independent samples ttest and one-way analysis of variance (ANOVA), and correlation analysis was performed by Pearson correlation analysis. Differences were considered statistically significant at $p \leq 0.05$. Correlation 

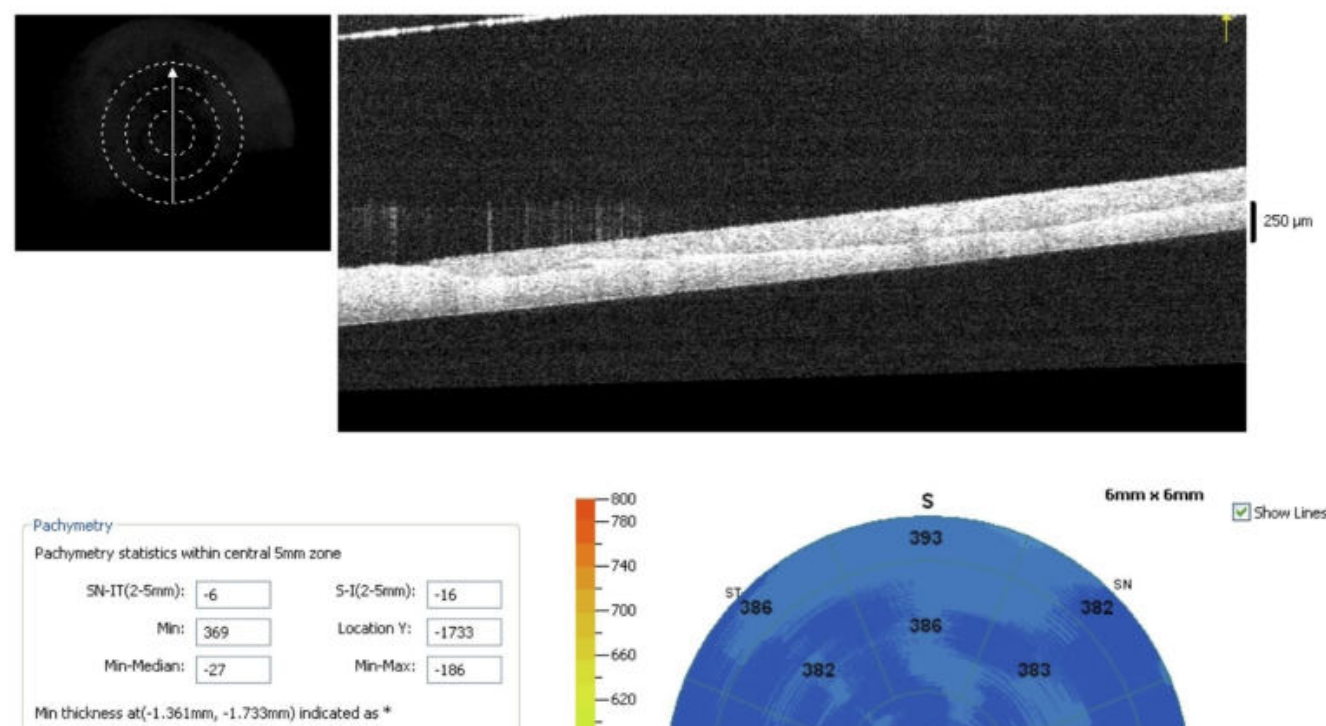

a)
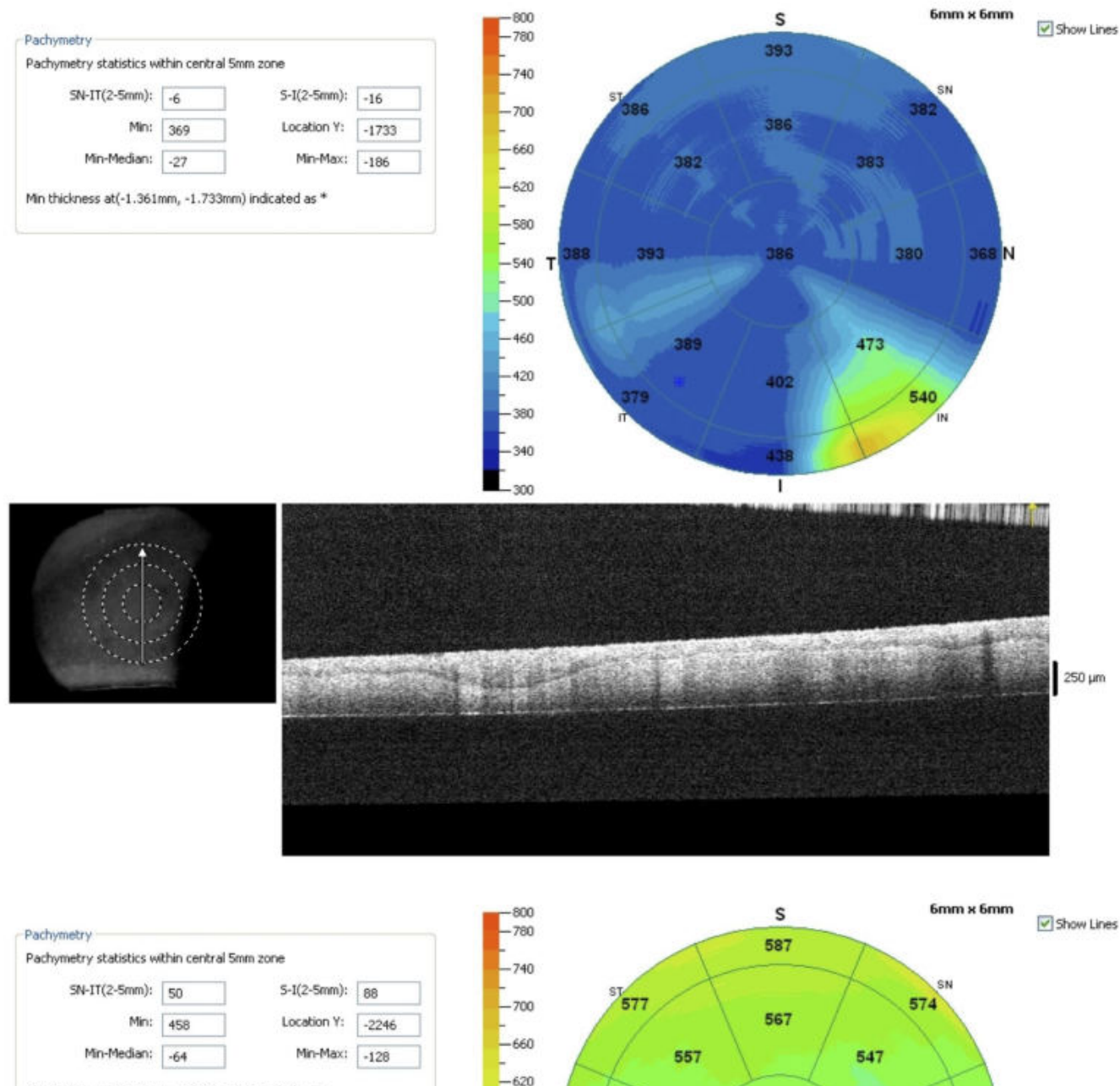

Mn thickness at $(0.784 \mathrm{~mm},-2.246 \mathrm{~mm})$ indicated as *

b)

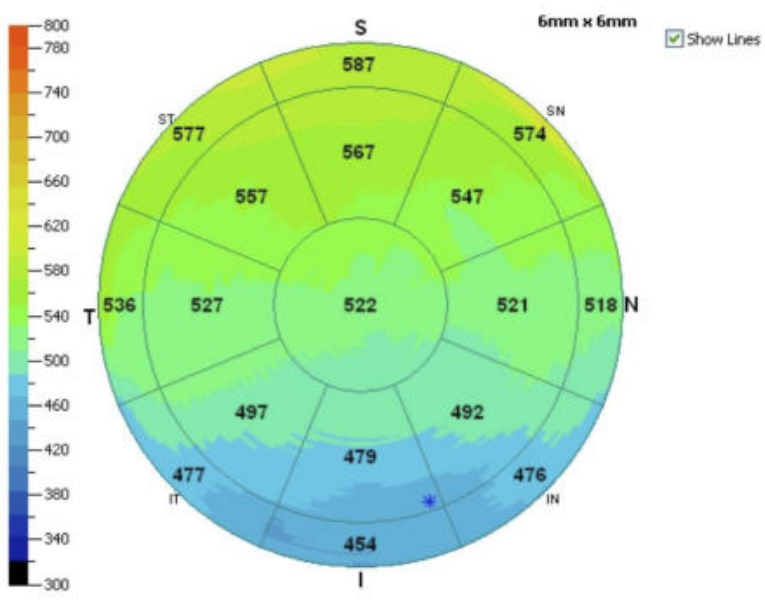

Fig. 2. Optical Coherence Tomography (OCT) images of L-PRF and A-PRF membranes: a) Thickness measurement of a LPRF membrane. Note irregular demarcation line at middle part of the membrane, b) Thickness measurement of an A-PRF membrane. Note irregular demarcation line at middle part of the membrane. 
coefficient $(r)$ showed a strong association between variables if it was higher than 0.5 .

\section{RESULTS}

\section{Demographics}

There were $5(50 \%)$ female and $5(50 \%)$ male subjects in PRF group, $10(100 \%)$ pregnant female subjects in amniotic membrane group. The mean ages of the subjects in PRF group and amniotic membrane group were 31 years (range between 20-40 years) and 28 years (range between $22-36$ years), respectively ( $p$ $=0.548)$.

\section{Modulus of Elasticity and Hardness}

In L-PRF group, mean modulus of elasticity value was calculated as $0.2 \pm 0.13 \mathrm{GPa}$ (range between 0.04 $0.5 \mathrm{GPa}$ ) while mean hardness value was computed $11.44 \pm 6.98 \mathrm{MPa}$ (range between 2.69-26.97 MPa). On the other hand,mean modulus of elasticity and hardness values in A-PRF group were calculated as $0.66 \pm 0.63 \mathrm{GPa}$ (range between 0.09-2.15 GPa) and $36.92 \pm 34.73 \mathrm{MPa}$ (range between 7.09-125.7 MPa), respectively. Differences for modulus of elasticity and hardness values between L-PRF and A-PRF groups were found statistically significant ( $p=0.03$, for both). In amniotic membrane group, mean modulus of elasticity value was calculated as $0.14 \pm 0.12 \mathrm{GPa}$ (range between 0.01-0.4 GPa) and found significantly lower than the L-PRF and A-PRF groups $(p=0.02)$. Moreover, mean hardness value in amniotic membrane group was calculated as $18.64 \pm 11.96 \mathrm{MPa}$ (range between 2.26-41.34 MPa) and it was found higher than L-PRF group and lower than A-PRF group $(p=0.06)$. The results of mechanical evaluation were summarized in Table. Statistically significant positive correlation between modulus of elasticity and hardness values for all groups were revealed. $(\mathrm{r}=0.95, p<$ 0.001).

\section{SD-OCT}

Mean thicknesses of the PRF membranes were obtained as $429.3 \pm 77.04 \mu \mathrm{m}$ (range between 345-543 $\mu \mathrm{m})$ and $566.1 \pm 146.71 \mu \mathrm{m}$ (range between 389-866 $\mu \mathrm{m})$ in L-PRF group and A-PRF group, respectively $(p=0.018)$ while the mean thickness of the amniotic membranes was calculated as $132.11 \pm 29.86 \mu \mathrm{m}$ (range between 92-189 $\mu \mathrm{m}$ ). Thickness measurements revealed that significantly lower values were recorded in L-PRF group when compared to A-PRF group ( $p=$ 0.018 ). Likewise, in amniotic membrane group mean thickness measurement was found significantly lower when compared to L-PRF and A-PRF groups ( $p<$ $0.001)$. Interestingly, an irregular demarcation line was observed approximately at middle part of the membranes probably due to the difference of light reflectivity in both L-PRF and A-PRF groups (Fig. 2).

\section{SEM}

Buffy coat sections of fibrin matrices were monitored by SEM analysis and cell behavior and cell contents were tried to be illuminated in the distal region of prepared samples. Scanning electron microscopyimages were obtained under the same magnifications and were shown in Fig. 3 comparatively. The fibrin structure and cellular locations of the L-PRF membranes were depicted in Fig. 3a and Fig. 3c, respectively while the A-PRF membranes were depicted in Fig. $3 b$ and Fig. 3d, respectively.

Fiber diameter was found in the range of $50 \mathrm{~nm}$ to $480 \mathrm{~nm}$ in L-PRF group, while it was in the range of $140 \mathrm{~nm}$ to $740 \mathrm{~nm}$ in A-PRF group. The mean value was calculated as $210 \pm 80 \mathrm{~nm}$ in L-PRF group, while it was computed as $390 \pm 160 \mathrm{~nm}$ in A-PRF group. It was observed that the diameters of the fibers formed in the structure of L-PRF membranes were smaller than the A-PRF membranes.

In the L-PRF group, cells were observed to be located on the surface of the formed fibrin structure. Contrary to this, cells were observed among the fibrin structure in the A-PRF group. These observations were shown in Fig. 4. The conducted Image $\mathrm{J}$ analysis revealed that the fiber size of the amniotic membrane was in the range of $30 \mathrm{~nm}$ to $140 \mathrm{~nm}$ and the mean value was calculated as $80 \pm 30 \mathrm{~nm}$.

\section{Macroscopic Observation}

Although same amount of blood sample was utilized for the obtaining of both membranes, mean length of L-PRF membranes was measured higher that of A-PRF membranes (Fig. 5). The mean length of the L-PRF membranes was measured as $3.32 \pm 0.13 \mathrm{~cm}$ (range between 3.2-3.5 cm), while the mean length of the A-PRF membranes was computed as $2.52 \pm 0.14$ 

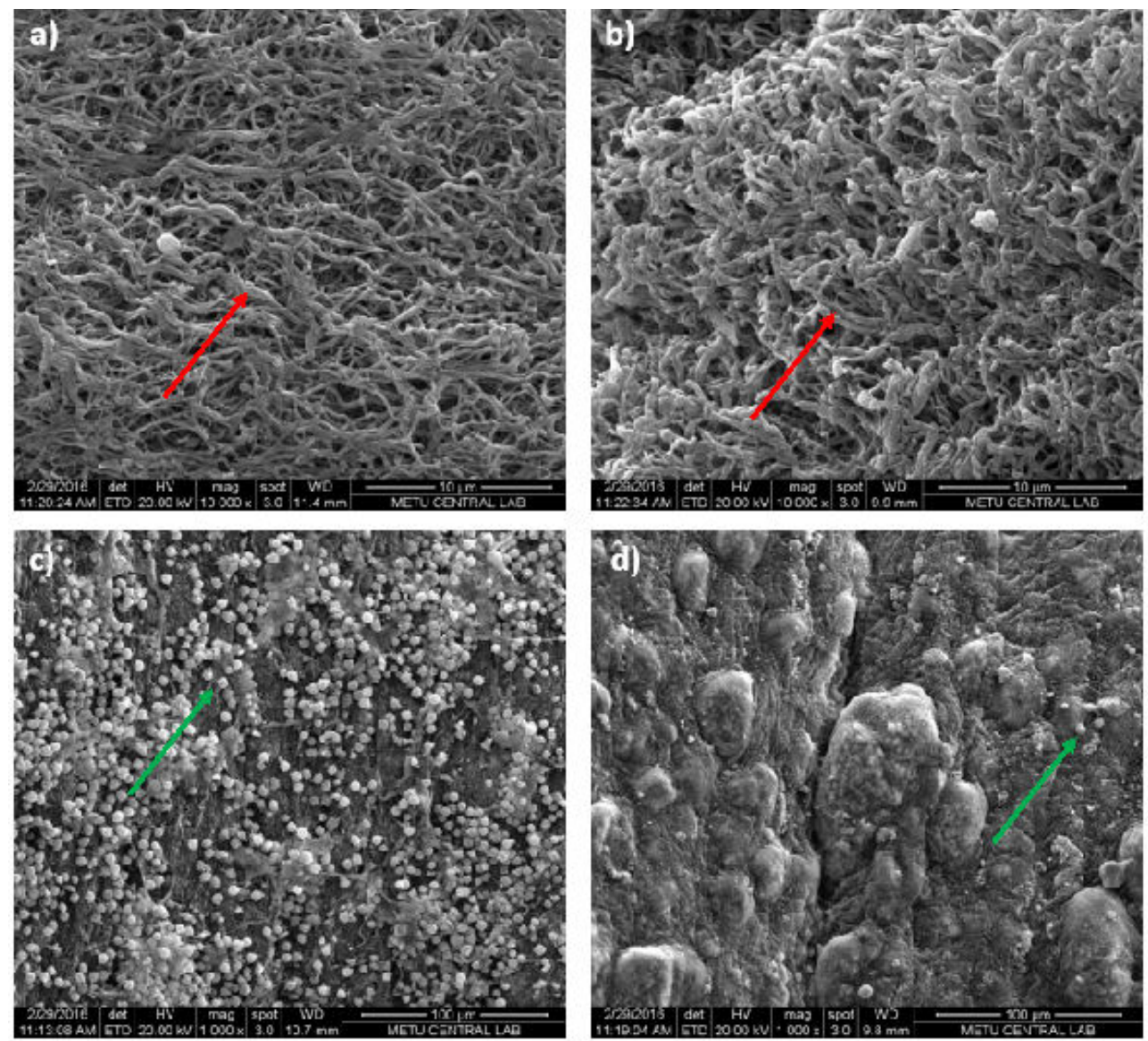

Fig. 3. Scanning Electron Microscopy (SEM) images of L-PRF and A-PRF membranes: a) Small-diameter fibrin fibers formed a dense 3D architecture with lower porosity and smaller pore diameters in L-PRF group, b) Larger diameter fibrin fibers formed a loose 3D architecture with higher porosity and larger pore diameters in A-PRF group, c) Small, round shape cells were observed to be located on the surface of the formed fibrin structure in L-PRF group, d) Small, round shape cells were observed among the fibrin structure in A-PRF group (Red arrows: fibers, green arrows: cells).
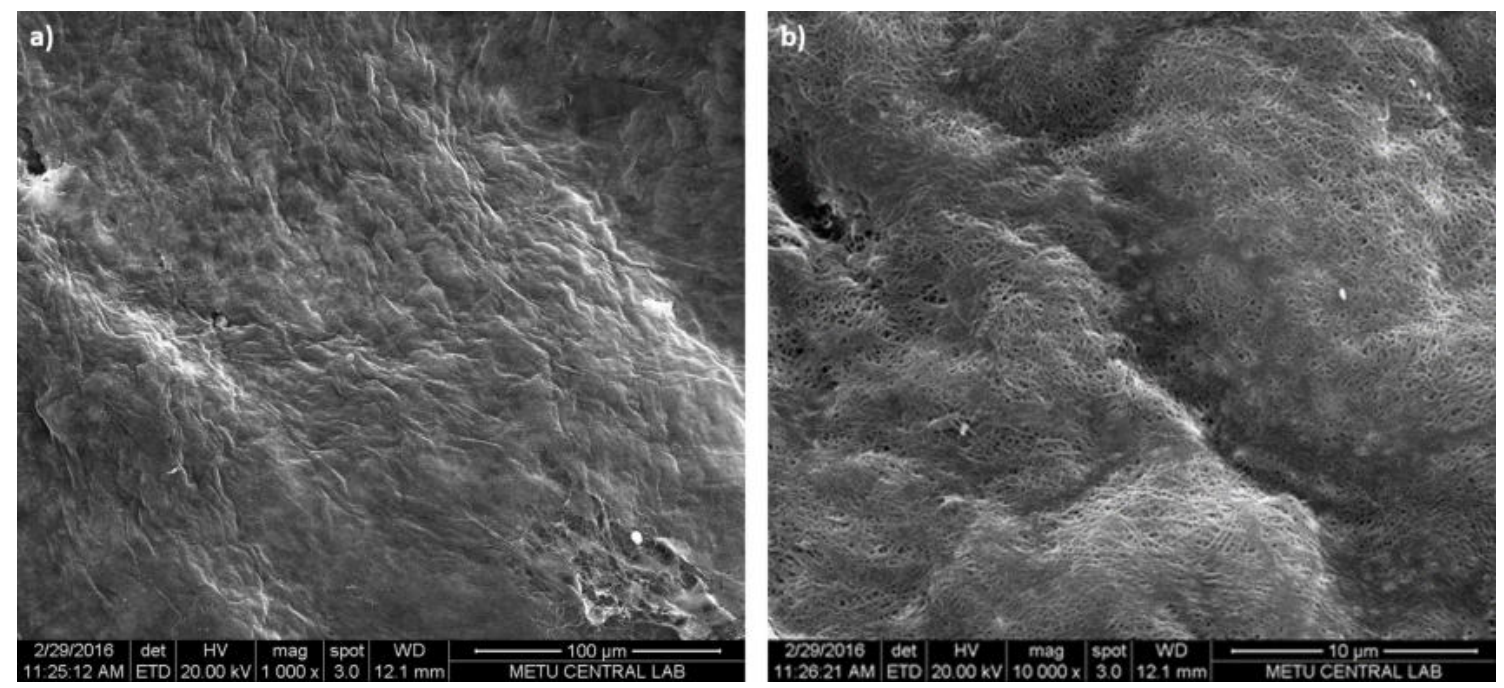

Fig. 4. Scanning Electron Microscopy (SEM) images of amniotic membranes: a) Surface topography of amniotic membrane, b) Thinner fibers firmly cross-matched and formed a stiff 3D architecture with lower porosity and smaller pore diameters in amniotic membrane group. 


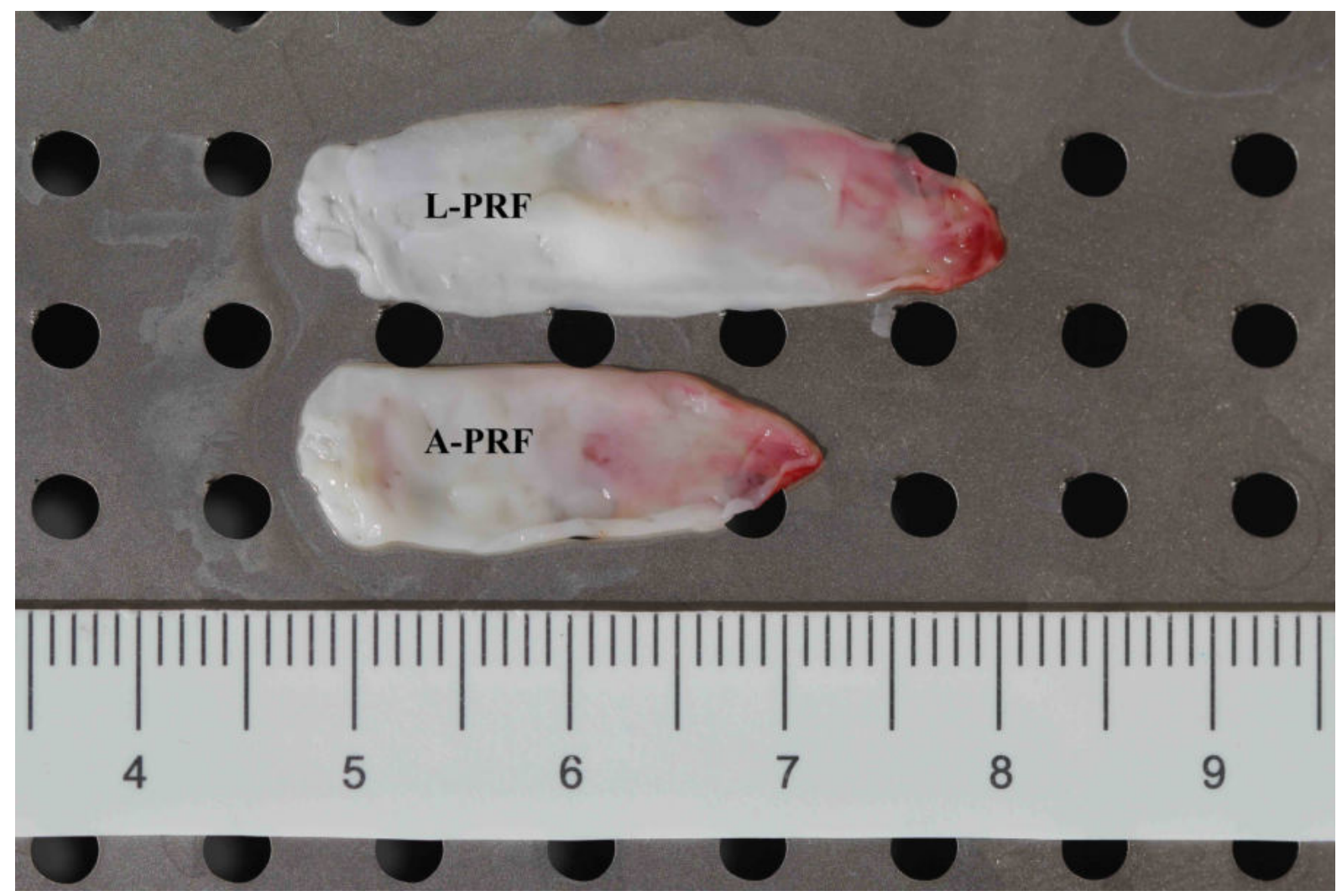

Fig. 5. Macroscopic appearance of L-PRF and A-PRF membranes after pressing.

\section{Degradation Results of the Samples}

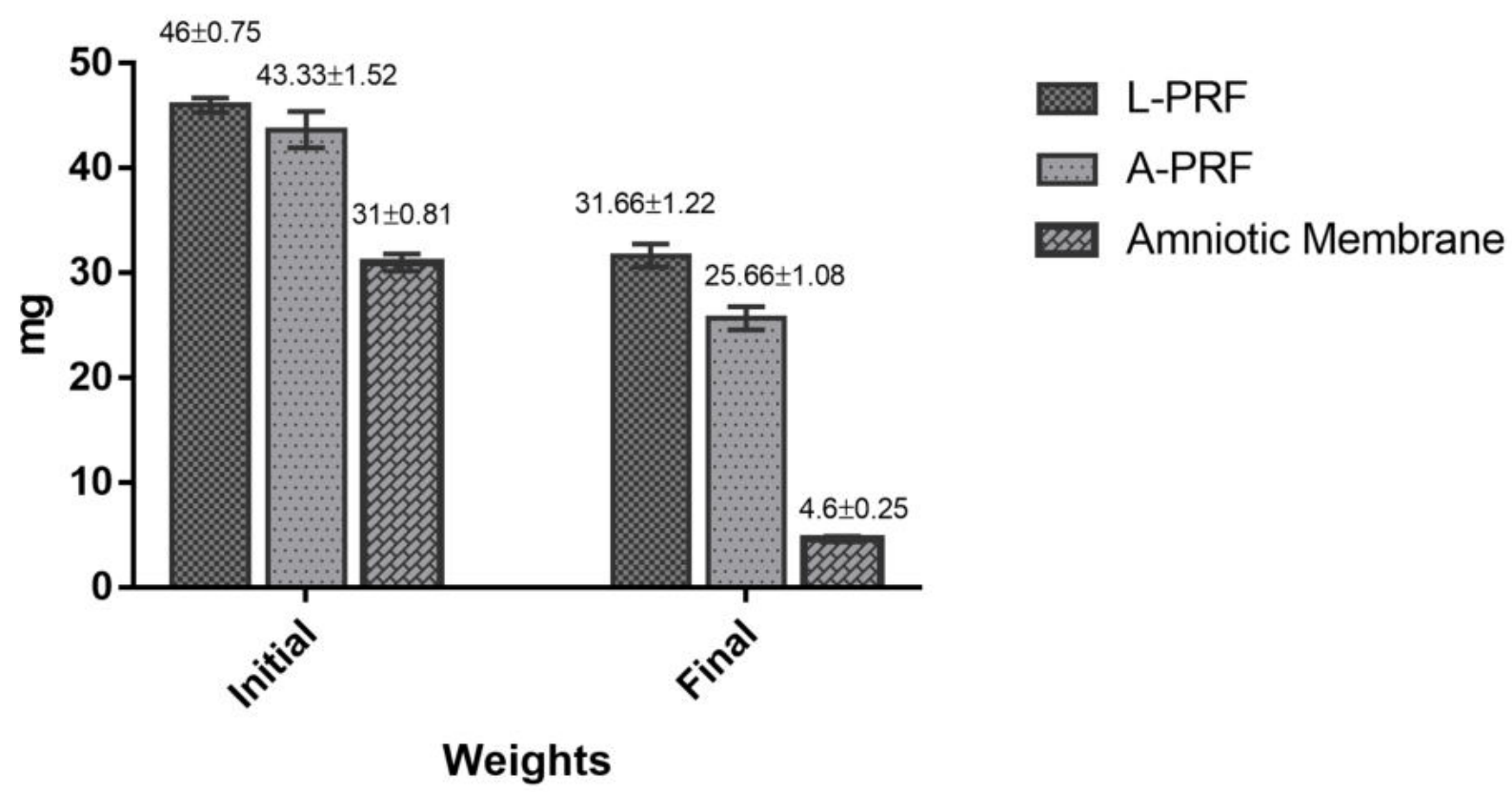

Fig. 6. Results of in vitro degradation test. 
$\mathrm{cm}$ (range between 2.3-2.7 $\mathrm{cm}$ ). The L-PRF membranes were found significantly longer than the A-PRF membranes $(p<0.001)$.

\section{In Vitro Degradation}

Degradation profiles of the membranes were depicted in Fig. 6. Advanced PRF membranes lost its $40.77 \% \pm 0.73$ of initial weight at the end of the in vitro shaking test, while L-PRF membranes lost $31.15 \% \pm 0.85$ of its initial weight. On the other hand, amniotic membranes degraded by $85.16 \% \pm 1.27$ at the end of the 1 week. These results showed thatamniotic membranes degraded remarkably faster than L-PRF and A-PRF membranes.

\section{DISCUSSION}

In recent years, studies have demonstrated that the use of platelet enriched fibrin products which are known to be a rich source of autologous chemokines and GFs, provide faster healing rates and enhanced recovery for tissue regeneration $[5,17-20]$. Choukroun's L-PRF is a second generation platelet concentrate and can be convertible into a wellorganized fibrin scaffold (L-PRF membrane) consisting of endogenous bio-signal molecules such as platelet-derived GF (PDGF), insulin-like GF (IGF), vascular endothelial GF (VEGF), and transforming growth factor-Beta (TGF- $\beta$ ), circulating stem cells, and proteins such as vitronectin, fibronectin, and Bone Morphogenetic Protein (BMP) [4-7]. These leucocyteand platelet-rich fibrin membranes are one of the convenient graft material among other members of fibrin family due to its facile production process and self-regeneration capacity [21]. Because of L-PRF's natural fibrin framework properties, GFs can keep their activity in fibrin mesh that allows for their progressive release over time (7-11 days) [22, 23]. The accumulation of these autogenous sources in an excellent 3D architecture therefore provides it desirable applications in clinical fields such as oral, ear-nose-throat, orthopedic, plastic, and ophthalmologic surgeries [13, 24-27]. However, different preparation protocols have been trending upward in order to obtain more vigorous biofunctional material. To this end, a new concept "A-PRF", has increasingly become popular since 2014
[9-11]. However, the aforementioned studies mostly focused on this novel architecture in terms of cellularity by histological examinations up to now [911], and there have been no studies showing mechanical and morphological properties of A-PRF that are important for surgeons during the operations.

Human amniotic membrane, the innermost layer of the placenta, is a multi-layered fetal membrane that is composed of a single epithelial layer, an avascular stroma and a thick basement membrane [28]. The epithelial cells are considered as an important source of anti-inflammatory, anti-scarring and anti-microbial bio-signals, and show advantages like stem cells for tissue regeneration [28-30]. It has been used for the reconstruction of skin, oral cavity, head and neck, bladder and ocular surface as a biostatic allograft [29, 31-36], and for the stem cell cultivation as afeeder layer [37]. However, some drawbacks such as complicated preparation process, transmission of infection, and requirement of special storage conditions set to $-800 \mathrm{C}$ may restrict its clinical use. In addition, variable biomechanical properties of amniotic membranes due to the variation of the components such as collagen, proteoglycan and elastin should be considered in the reconstruction of large and deep tissue defects in the clinical field [38].

In the present study, three different natural biomaterials were compared in terms of mechanical strength by nano-indentation, morphological characteristics by OCT and SEM, and degradation profile by in vitro degradation test. The nanoindentation test revealed significantly higher modulus of elasticity and hardness values in A-PRF group when compared to L-PRF and amniotic membrane groups. A study of Sam et al. [16] investigated mechanical properties of L-PRF membrane by a triboindenter (T1 950, Hysitron Nanotechnology, US) revealed that the modulus of elasticity and hardness values of L-PRF membrane were $0.35 \mathrm{GPa}$ and $10.67 \mathrm{MPa}$, respectively. In consistence with the findings of this study[16] we demonstrated that the modulus of elasticity and hardness values of L-PRF membrane were $0.2 \mathrm{GPa}$ and $11.44 \mathrm{MPa}$, respectively. Moreover, a recent study by Khorshidi et al. [15] evaluated the tensile strength, stiffness, and toughness of the early L-PRF membranes and compared it with PRGF/Endoret membranes which can be obtained by a multi-step process. They demonstrated that early L- 
PRFmembranes had stronger mechanical properties and might have easier clinical handling as a scaffold in periodontal regenerative procedures.

One of the most importantfindings of the present work was that changing the centrifugation force leads to significantly different fiber diameter and configuration in micro-architecture of the PRF membranes. In SEM analysis, the major finding was that the fiber diameters of A-PRF membranes measured from the buffy coat area by Image-J analysis were remarkably higher than those of the L-PRF membranes. Additionally, larger diameter fibers formeda loose 3D architecture with higher and larger diameter pores. These findings could be related to the lower centrifugal speed (1,500 rpm) in A-PRF group. Because of the high centrifugal speed $(2,700 \mathrm{rpm})$ in L-PRF group, more intense fibrin fragmentation occurs and small-diameter fibers are formed firmly which results in dense and thin structure with lower porosity. Furthermore, the OCT analysis revealed significantly higher thickness measurements for APRF membranes when compared to L-PRF membranes. It was thought that loosely cross-matched thicker fibers in A-PRF membranes configure thicker membranes. Additionally, the macroscopic sizes of APRF membranes were remarkably smaller than the L-PRF membranes. It can be speculated that higher centrifugal speed for L-PRF procedure followed by pressing in order to obtain L-PRF membranes results in thinner fibrin fibers condensed into a more stiff and dense structure with small pore sizes. In contrast, lower centrifugal speed for A-PRF procedure protects fibrin fibers from disruption and induces the formation of thicker membranes, which cannot be easily pressed. Consequently, we obtained small and thick A-PRF membranes and large and thin L-PRF membranes by altering the centrifugal force. The aforementioned study by Sam et al. [16] investigated the surface morphology and cell types of L-PRF membranes by SEM and revealed that dense clusters of platelets which are resulted from extensive aggregation and clotting were resting on a mature fibrin structure. In a previous study by Hatakeyama et al .[39], investigators evaluated the ultra-structural morphology of the L-PRF membranes and demonstrated a more highly condensed fibrin fiber network that was regularly arranged. In conclusion, the analysis of previous literature studies clearly shows that the buffy coat area of the L-PRF membrane is made up of thick fibers which make it a dense material.

To the best of our knowledge, no research to date has focused on the mechanical properties of PRF despite its clinical use for over a decade [40], and this is the first experimental study investigating the mechanical and morphological properties of A-PRF membrane. The results of the present work demonstrated that the A-PRF membranes exhibit higher modulus of elasticity and hardness values. It is our opinion that higher fibrin diameter within the APRF structure might provide enhanced mechanical strength to this material. As far as we know, this is the first attempt also to evaluate the thickness of the PRF membranes with OCT. We demonstrated that A-PRF membranes exhibit higher thickness measurements that can be linked with the increased fiber diameter within the structure. As a result, it can be interpreted that sparsely located thick fibrin strands within the structure of A-PRF lead to thick membrane formation and provide higher mechanical strength. Interestingly, an irregular demarcation line was observed approximately in the middle part of the all membranes in both L-PRF and A-PRF groups. We speculated that, it is due to the difference of light reflectivity originated from variation of cellular density in upper and lower part of the PRF membranes.

As an evaluation of cellular locations in L-PRF and A-PRF membranes, we visualized the distal part of both membranes (neighboring red blood cells) under the same magnifications. In contrast with the findings of the study reported by Hatakeyama et al. [39] platelets seemed to be located on the surface of the formed fibrin structure in the L-PRF group. Contrary to this, cells are observed among the fibrin structure in the A-PRF group. These findings were exhibited correlation with our SEM results mentioned above. Here, it can be speculated that the high centrifugal speed might cause dense and firmly adhered fibrin architecture that might force the cells to migrate to the surface of the membrane. On the other hand, cells were thought to be hold into the sparse fibrin structure with more inter-fibrous space in A-PRF group. Although the study of Hatakeyama et al. [39] showed that the platelet concentrates were embedded within the highly condensed fibrin fibers in L-PRF group, our results were found distinct from 
their analysis by means of applied centrifugal force. Yet, it needs to be thoroughly determined whether the centrifugal force is the only important indicative for cell distribution within the fibrin architecture. It was suggested that further studies need to be performed to address this issue.

The present study basically demonstrates that the lower fiber disintegration together with lower cellular scattering were succeeded by means of lower centrifugal force in A-PRF group. Therefore, it was also refrained from cell death due to the cell scattering. Additionally, thicker fibers together with more interfibrous space in A-PRF group might provide the cellular integrity within the $3 \mathrm{D}$ structure. On the other hand, this study did not evaluate the cellular types of PRF membranes with histological analysis. However, the morphological results might be related to previous studies in terms of protected cellular sources within the fibrin meshesthat provide higher and longer GF release $[9,10]$. Cellular distribution, penetration and allocation were compared between the L-PRF and APRF in a study presented by Ghanaati et al. [9] and it was shown that the penetration of T-lymphocytes, CD34+ stem cells, macrophages and neutrophilic granulocytes in A-PRF group was deeper than the LPRF group resulting the higher GF intensity within the structure.

Another important finding of our study was that the change in degradation profile of the PRF membranes depending on the centrifugal force. The degradation of L-PRF membranes was approximately $10 \%$ lower than the A-PRF membranes at the end of 1 -week period. These results could be explained by the formation of more close and robust fibrin strands consisting of small inter-fibrous space in L-PRF group that restrict the structure against PBS infiltration which led to fiber disintegration. However, the degradation rate of both membranes was comparable in terms of maintaining its initial physical features up to 5 days. Moreover, remaining membranes after 1 week in the surgical side might continue to provide mechanical and chemical support to the wound gap.

\section{CONCLUSION}

In conclusion, it would be more advantageous to apply more practical graft materials to achieve faster, innocuous and cost-effective treatments in regenerative medicine. A-PRF, a novel tissue engineered product, is a temporary matrix protein consisting GFs and stem cells that have a pivotal role in wound healing. A-PRF might be able to promote cell growth and migration in order to achieve acceleration of wound healing while promoting vascularization of tissue gap. Although this study mostly focused on the advantages of the A-PRF membranes, the applicability of this new concept has to be proven through clinical studies such as pterygium surgery, scleral regeneration, corneal repair and orbital socket reconstruction. On the other hand, this study was intended to guide surgeons in the selection of PRF membranes, since morphological and mechanical properties of the graft materials are discussed in detail here.

\section{Conflict of interest}

The authors disclosed no conflict of interest during the preparation or publication of this manuscript.

\section{Financing}

The authors disclosed that they did not receive any grant during conduction or writing of this study.

\section{Acknowledgements}

This work was supported partially by funds from the BMT Calsis Co., Ankara, Turkey. The authors would like to thank Zafer Artvin and Arda Buyuksungur for their experimental help and advice.

\section{REFERENCES}

1. Lin X, Huang J, Shi Y, Liu W. Tissue engineering and regenerative medicine in applied research: a year in review of 2014. Tissue Eng Part B Rev 2015;21:177-86.

2. Burnouf T, Goubran HA, Chen T-M, Ou K-L, El-Ekiaby M, Radosevic M. Blood-derived biomaterials and platelet growth factors in regenerative medicine. Blood Rev 2013;27:77-89.

3. Anitua E, Sánchez M, Orive G, Andía I. The potential impact of the preparation rich in growth factors (PRGF) in different medical fields. Biomaterials 2007;28:4551-60.

4. Dohan Ehrenfest DM, Rasmusson L, Albrektsson T. Classification of platelet concentrates: from pure platelet-rich plasma (P-PRP) to leucocyte- and platelet-rich fibrin (L-PRF). Trends Biotechnol 2009;27:158-67.

5. Kang Y-H, Jeon SH, Park J-Y, Chung J-H, Choung Y-H, Choung $\mathrm{H}-\mathrm{W}$, et al. Platelet-rich fibrin is a Bioscaffold and 
reservoir of growth factors for tissue regeneration. Tissue Eng Part A 2011;17:349-59.

6. Dohan DM, Choukroun J, Diss A, Dohan SL, Dohan AJJJ, Mouhyi J, et al. Platelet-rich fibrin (PRF): a second-generation platelet concentrate. Part I: technological concepts and evolution. Oral Surg Oral Med Oral Pathol Oral Radiol Endod 2006;101:3744.

7. Dohan DM, Choukroun J, Diss A, Dohan SL, Dohan AJJJ, Mouhyi J, et al. Platelet-rich fibrin (PRF): a second-generation platelet concentrate. Part II: Platelet-related biologic features. Oral Surg Oral Med Oral Pathol Oral Radiol Endod 2006;101:4550 .

8. Sadeghi-Ataabadi M, Mostafavi-Pour Z, Vojdani Z, Sani M, Latifi M, Talaei-Khozani T. Fabrication and characterization of platelet-rich plasma scaffolds for tissue engineering applications. Mater Sci Eng C Mater Biol Appl 2017;71:372-80.

9. Ghanaati S, Booms P, Orlowska A, Kubesch A, Lorenz J, Rutkowski J, et al. Advanced platelet-rich fibrin: a new concept for cell-based tissue engineering by means of inflammatory cells. J Oral Implantol 2014;40:679-89.

10. Kobayashi E, Flückiger L, Fujioka-Kobayashi M, Sawada K, Sculean A, Schaller B, et al. Comparative release of growth factors from PRP, PRF, and advanced-PRF. Clin Oral Investig 2016;20:2353-60.

11. Dohan Ehrenfest DM, Pintyo NR, Pereda A, Jimenez P, Del Corso M, Kang BS, et al. The impact of the centrifuge characteristics and centrifugation protocols on the cells, growth factors and fibrin architecture of a leukocyte- and platelet-rich fibrin (L-PRF) clot and membrane. Platelets 2018;29:171-84.

12. Can ME, Çakmak HB, Dereli Can G, Ünverdi H, Toklu Y, Hücemenoğlu S. A novel technique for conjunctivoplasty in a rabbit model: platelet-rich fibrin membrane grafting. J Ophthalmol 2016;2016:1965720.

13. Can ME, Dereli Can G, Cagil N, Cakmak HB, Sungu N. Urgent therapeutic grafting of platelet-rich fibrin membrane in descemetocele. Cornea 2016;35:1245-9.

14. Dohan Ehrenfest DM, Del Corso M, Diss A, Mouhyi J, Charrier J-B. Three-dimensional architecture and cell composition of a Choukroun's platelet-rich fibrin clot and membrane. J Periodontol 2010;81:546-55.

15. Khorshidi H, Raoofi S, Bagheri R, Banihashemi H. Comparison of the Mechanical Properties of Early Leukocyteand Platelet-Rich Fibrin versus PRGF/Endoret Membranes. Int J Dent 2016;2016:1849207.

16. Sam G, Vadakkekuttical RJ, Amol NV. In vitro evaluation of mechanical properties of platelet-rich fibrin membrane and scanning electron microscopic examination of its surface characteristics. J Indian Soc Periodontol 2015;19:32-6.

17. Dohan Ehrenfest DM, Del Corso M, Inchingolo F, Sammartino G, Charrier J-B. Platelet-rich plasma (PRP) and platelet-rich fibrin (PRF) in human cell cultures: growth factor release and contradictory results. Oral Surg Oral Med Oral Pathol Oral Radiol Endod 2010;110:418-21.

18. Fontana S, Olmedo DG, Linares JA, Guglielmotti MB, Crosa ME. Effect of platelet-rich plasma on the peri-implant bone response: an experimental study. Implant Dent 2004;13:73-8.

19. Sharma A, Pradeep AR. Autologous platelet-rich fibrin in the treatment of mandibular degree II furcation defects: a randomized clinical trial. J Periodontol 2011;82:1396-403.

20. Simonpieri A, Choukroun J, Del Corso M, Sammartino G, Dohan Ehrenfest DM. Simultaneous sinus-lift and implantation using microthreaded implants and leukocyte- and platelet-rich fibrin as sole grafting material: a six-year experience. Implant Dent 2011;20:2-12.

21. Ji B, Sheng L, Chen G, Guo S, Xie L, Yang B, et al. The combination use of platelet-rich fibrin and treated dentin matrix for tooth root regeneration by cell homing. Tissue Eng Part A 2015;21:26-34.

22. Dohan Ehrenfest DM, Bielecki T, Jimbo R, Barbé G, Del Corso $\mathrm{M}$, Inchingolo $\mathrm{F}$, et al. Do the fibrin architecture and leukocyte content influence the growth factor release of platelet concentrates? An evidence-based answer comparing a pure platelet-rich plasma (P-PRP) gel and a leukocyte- and plateletrich fibrin (L-PRF). Curr Pharm Biotechnol 2012;13:1145-52.

23. Dohan Ehrenfest DM, Diss A, Odin G, Doglioli P, Hippolyte M-P, Charrier J-B. In vitro effects of Choukroun's PRF (plateletrich fibrin) on human gingival fibroblasts, dermal prekeratinocytes, preadipocytes, and maxillofacial osteoblasts in primary cultures. Oral Surg Oral Med Oral Pathol Oral Radiol Endod 2009;108:341-52.

24. Bielecki T, Dohan Ehrenfest DM. Platelet-rich plasma (PRP) and Platelet-Rich Fibrin (PRF): surgical adjuvants, preparations for in situ regenerative medicine and tools for tissue engineering. Curr Pharm Biotechnol 2012;13:1121-30.

25. Dohan Ehrenfest DM, Andia I, Zumstein MA, Zhang C-Q, Pinto NR, Bielecki T. Classification of platelet concentrates (Platelet-Rich Plasma-PRP, Platelet-Rich Fibrin-PRF) for topical and infiltrative use in orthopedic and sports medicine: current consensus, clinical implications and perspectives. Muscles Ligaments Tendons J 2014;4:3-9.

26. Cieslik-Bielecka A, Choukroun J, Odin G, Dohan Ehrenfest DM. L-PRP/L-PRF in esthetic plastic surgery, regenerative medicine of the skin and chronic wounds. Curr Pharm Biotechnol 2012;13:1266-77.

27. Braccini F, Tardivet L, Dohan Ehrenfest DM. [The relevance of Choukroun's Platelet-Rich Fibrin (PRF) during middle ear surgery: preliminary results]. Rev Laryngol Otol Rhinol (Bord) 2009;130:175-80. [Article in French]

28. Litwiniuk M, Grzela T. Amniotic membrane: new concepts for an old dressing. Wound Repair Regen 2014;22:451-6.

29. Zhao B, Liu J-Q, Zheng Z, Zhang J, Wang S-Y, Han S-C, et al. Human amniotic epithelial stem cells promote wound healing by facilitating migration and proliferation of keratinocytes via ERK, JNK and AKT signaling pathways. Cell Tissue Res 2016;365:85-99.

30. Russo V, Tammaro L, Di Marcantonio L, Sorrentino A, Ancora M, Valbonetti L, et al. Amniotic epithelial stem cell biocompatibility for electrospun poly(lactide-co-glycolide), poly( $\varepsilon$-caprolactone), poly(lactic acid) scaffolds. Mater Sci Eng C Mater Biol Appl 2016;69:321-9.

31. Mohammadi AA, Johari HG, Eskandari S. Effect of amniotic membrane on graft take in extremity burns. Burns 2013;39:113741.

32. Arai N, Tsuno H, Okabe M, Yoshida T, Koike C, Noguchi M, 
et al. Clinical application of a hyperdry amniotic membrane on surgical defects of the oral mucosa. J Oral Maxillofac Surg 2012;70:2221-8.

33. Kesting MR, Wolff K-D, Nobis CP, Rohleder NH. Amniotic membrane in oral and maxillofacial surgery. Oral Maxillofac Surg 2014;18:153-64.

34. Jerman UD, Veranič P, Kreft ME. Amniotic membrane scaffolds enable the development of tissue-engineered urothelium with molecular and ultrastructural properties comparable to that of native urothelium. Tissue Eng Part C Methods 2014;20:31727.

35. Malhotra C, Jain AK. Human amniotic membrane transplantation: different modalities of its use in ophthalmology. World J Transplant 2014;4:111-21.

36. Mamede AC, Carvalho MJ, Abrantes AM, Laranjo M, Maia

CJ, Botelho MF. Amniotic membrane: from structure and functions to clinical applications. Cell Tissue Res 2012;349:44758.

37. Lai J-Y. Photo-cross-linking of amniotic membranes for limbal epithelial cell cultivation. Mater Sci Eng C Mater Biol Appl 2014;45:313-9.

38. Lai J-Y. Carbodiimide cross-linking of amniotic membranes in the presence of amino acid bridges. Mater Sci Eng C Mater Biol Appl 2015;51:28-36.

39. Hatakeyama I, Marukawa E, Takahashi Y, Omura K. Effects of platelet-poor plasma, platelet-rich plasma, and platelet-rich fibrin on healing of extraction sockets with buccal dehiscence in dogs. Tissue Eng Part A 2014;20:874-82.

40. Miron RJ, Fujioka-Kobayashi M, Bishara M, Zhang Y, Hernandez M, Choukroun J. Platelet-rich fibrin and soft tissue wound healing: a systematic review. Tissue Eng Part B Rev 2017;23:83-99. 